\title{
The Sniffing Bead System as a Useful Diagnostic Tool for Olfactory Dysfunction in COVID-19
}

\author{
Hyun Jin Min \\ Department of Otorhinolaryngology-Head and Neck Surgery, Chung-Ang University College of Medicine, Seoul, Korea \\ 코로나 환자에서의 후각 이상 증상을 감별하기 위한 선별검사 방법의 소개 \\ 민현진 \\ 중앙대학교 의과대학 이비인후과학교실
}

\author{
Received April 29, 2021 \\ Revised June 9, 2021 \\ Accepted June 9, 2021 \\ Address for correspondence \\ Hyun Jin Min, MD, PhD \\ Department of Otorhinolaryngology- \\ Head and Neck Surgery, \\ Chung-Ang University \\ College of Medicine \\ 84 Heukseok-ro, Dongjak-gu, \\ Seoul 06974, Korea \\ Tel +82-2-6299-1765 \\ Fax +82-2-825-1765 \\ E-mail jjinient@cau.ac.kr
}

\begin{abstract}
We present a sniffing bead system used to diagnose olfactory dysfunction in coronavirus disease 2019 (COVID-19) patients. A 25-year-old male presented with the loss of olfaction one week after he was diagnosed with severe acute respiratory syndrome coronavirus 2 (SARSCoV-2). He had no other symptoms such as fever or myalgia but only showed an absence of respiratory distress. Nasal endoscopy and paranasal sinus CT showed that the patient had no bilateral sinus diseases; cranial nerve MRI showed no abnormal signal intensity or enhancement. A sniffing bead system was applied using 2-phenylethyl alcohol for the objective assessment of olfactory dysfunction to confirm the presence of anosmia. Anosmia was diagnosed early by objective evaluation using a sniffing bead system and early intervention with olfactory training. This case report suggests that a verified, one-off system for objective measurement of olfactory dysfunction in COVID-19 with olfactory training in patients could facilitate the recovery of olfactory function. Korean J Otorhinolaryngol-Head Neck Surg 2022;65(2):107-11
\end{abstract}

Keywords COVID-19; Diagnosis; Olfactory dysfunction.

\section{Introduction}

The coronavirus disease 2019 (COVID-19) pandemic caused by severe acute respiratory syndrome coronavirus 2 (SARS$\mathrm{CoV}-2)$ remains a global health crisis. Reports suggest that the clinical features of COVID-19 range from asymptomatic infection to severe pneumonia and multiple organ dysfunction. ${ }^{1)}$ Olfactory dysfunction has been recently recognized as a common symptom of COVID-19, with a prevalence of up to $85.6 \%{ }^{2,3)}$ Here, we report a case with anosmia as the only symptom of COVID-19, confirmed by a sniffing bead system, a simple one-off olfactory threshold detection tool. The patient underwent olfactory training with the resolution of olfactory

This is an Open Access article distributed under the terms of the Creative Commons Attribution Non-Commercial License (https://creativecommons.org/licenses/by-nc/4.0) which permits unrestricted non-commercial use, distribution, and reproduction in any medium, provided the original work is properly cited. symptoms after three months. Although the prevalence of olfactory dysfunction has been widely reported, there are few tools for its objective measurement. In this case report, we introduce our sniffing bead system, which is useful for the objective measurement of olfactory dysfunction in COVID-19 without the risk of disease spread. It could lead to early diagnosis and intervention.

\section{Case}

A 25-year-old male with olfactory loss presented to our department. The patient's medical history included possible exposure to SARS-CoV-2, as he was diagnosed with COVID-19 soon after arriving from a foreign country. The patient had no underlying medical conditions such as hypertension, diabetes, or allergy; he had no fever or myalgia and showed an ab- 
sence of respiratory distress. He did not require medical therapy except preventive quarantine for two weeks.

One week after the COVID-19 diagnosis, he realized that he had lost his sense of smell. CT showed no bilateral sinus disease, except a retention cyst in the right maxillary sinus, and opacity in bilateral olfactory cleft (Fig. 1). Cranial nerve MRI was performed at the Department of Neurology and showed no abnormal signal intensity or enhancement in the cranial nerve within the scanned range (Fig. 2). Subjectively, the discomfort associated with olfactory loss received a score of zero
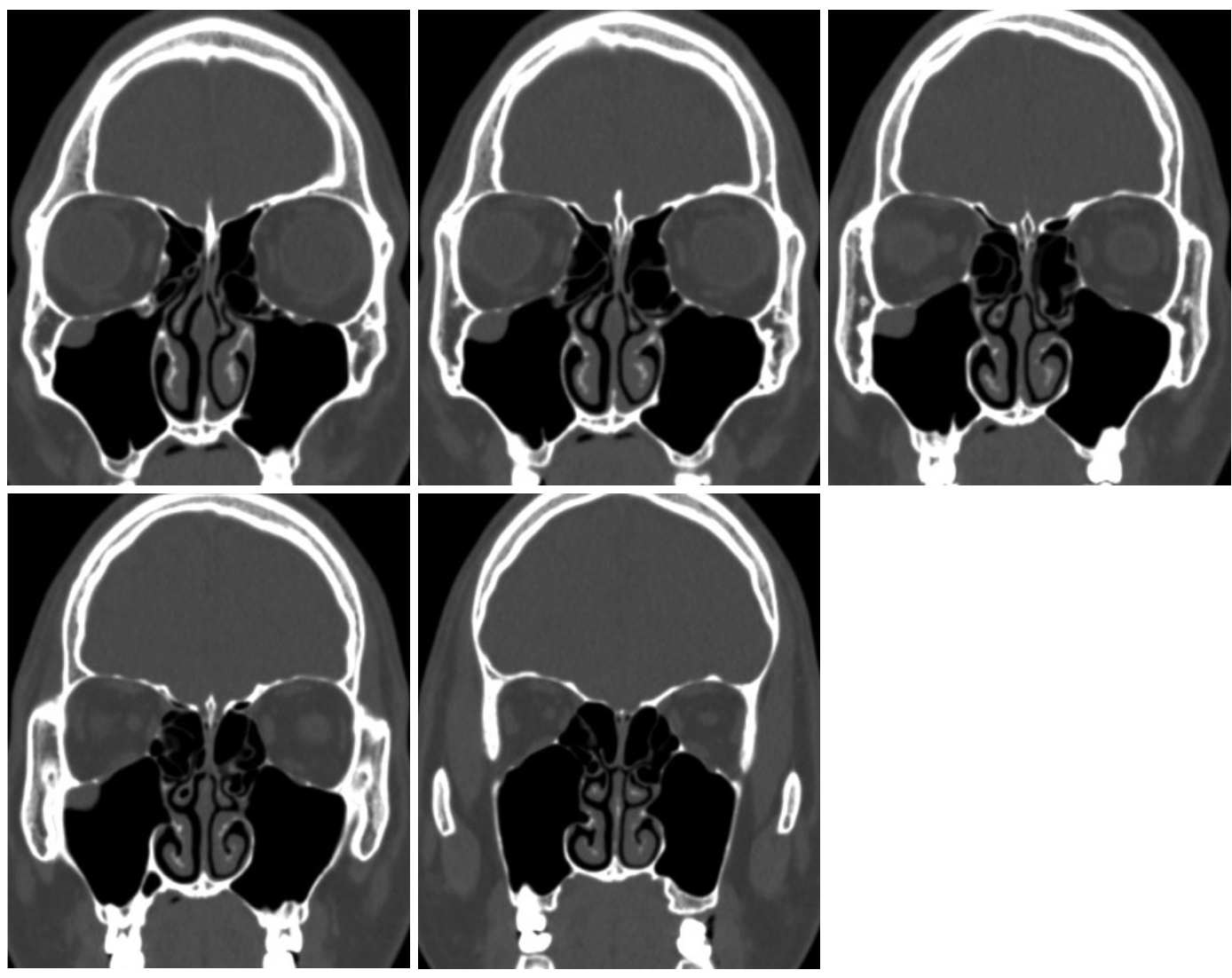

Fig. 1. Coronal images of paranasal sinus by CT. Paranasal CT images demonstrate partial soft tissue density in right olfactory cleft and total opacity in left olfactory cleft (from anterior to posterior ethmoid sinus level).
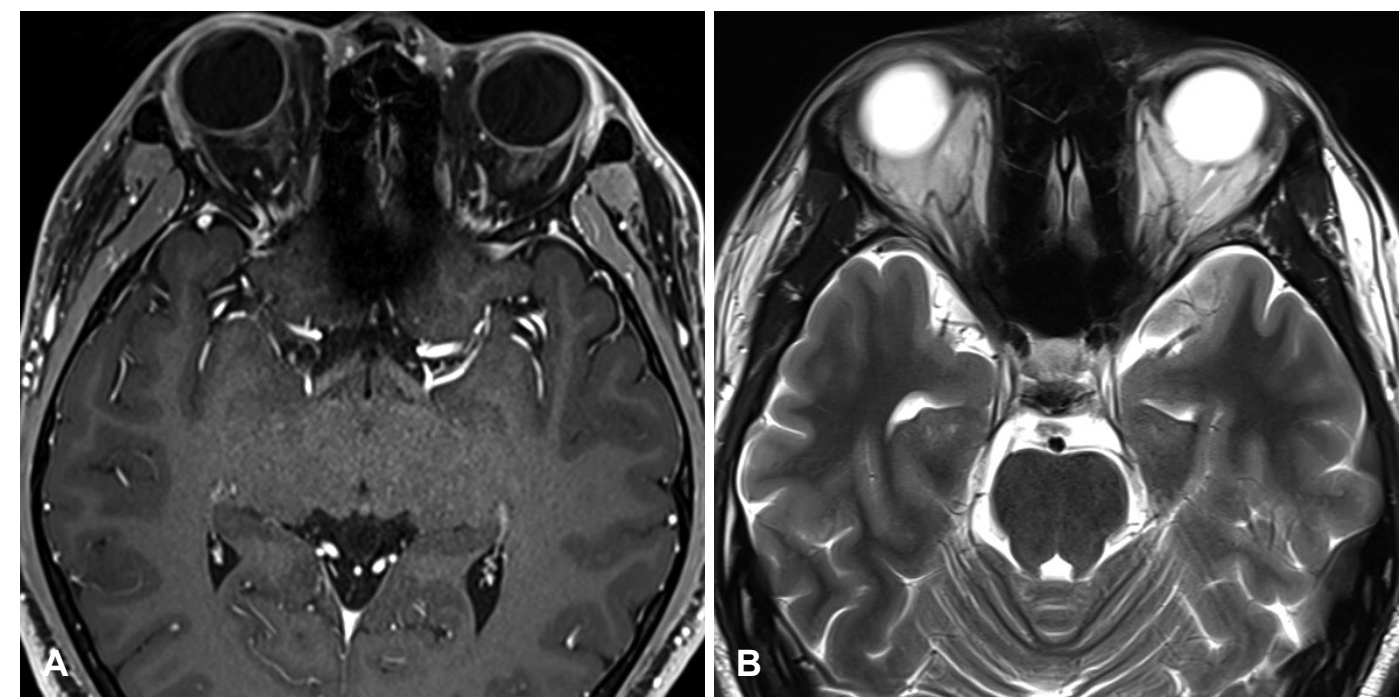

Fig. 2. Axial images of cranial nerve MRI. (A) T1-weighted enhanced and (B) T2-weighted axial images of the cranial nerve demonstrate no abnormal signal intensity or enhancement in olfactory bulb. 
on the visual analog scale. ${ }^{4}$

Conventional olfactory function tests that involve the use of odor-impregnated felt-tip pens cannot be applied to COVID-19 patients due to the possibility of disease spread. As this patient showed a continuous positive result for the COVID-19 test, we decided to perform a sniffing bead test, which was previously developed for screening olfactory dysfunction in geriatrics. ${ }^{5)}$ Unlike the conventional olfactory function test performed in our country, this is a one-off system, and any odor familiar to the patient can be packed into the beads. The sniffing bead system consists of several small beads $(7 \mathrm{~mm}$ in diameter) and a handpiece composed of a disposable plastic capsule that releases the odor after bead insertion (Fig. 3). ${ }^{5}$ The beads containing the odorants are intended for a single use, and the handpiece is wrapped in plastic vinyl. Therefore, we concluded that the sniffing bead system could be used for COVID-19 patients without the potential risk of virus spread. In this case, we used beads packed with 2-phenylethyl alcohol (PEA), as the odor was familiar within our population. The test was performed at out-patient clinic room by ENT specialist with protective clothing. The beads are placed and burst at $2 \mathrm{~cm}$ in front of both nostrils, and the patient was then asked if he recognized its fragrance. Diagnostic mean value of PEA threshold has been reported as $4.6 \pm 2.3$ for anosmia, $3.3 \pm 2.2$ for hyposmia, and $1.7 \pm 1.2$ for anosmia. ${ }^{6}$ The mean

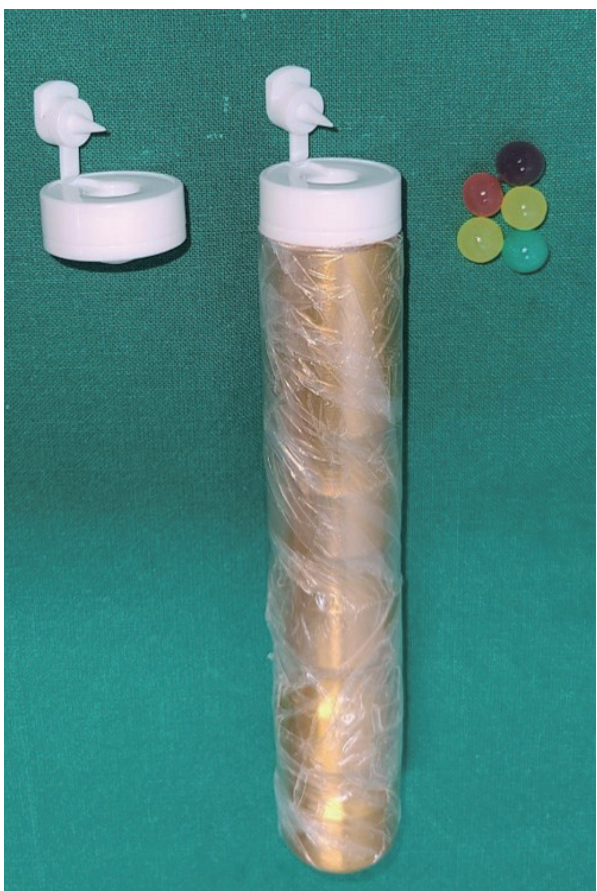

Fig. 3. Sniffing bead system applied to our COVID-19 patient for evaluation of olfactory dysfunction. Sniffing bead system is composed of small beads which contains odorants and a handpiece. value of the patient was 0 after repetition of three time, and could not detect the scent of PEA even at the highest concentration (10\% of PEA in distilled water). He was diagnosed with anosmia associated with COVID-19 and received olfactory training without the administration of systemic steroids.

Until two months after olfactory training, he did not notice any change in his olfactory function, either in terms of subjective sensation or the sniffing bead system test. However, after nine weeks, he noticed an improvement in symptoms and could identify the smell of PEA. After three months, his olfactory function improved, and the patient experienced no discomfort in daily life.

\section{Discussion}

Although the pathophysiology of olfactory dysfunction in COVID-19 remains unclear, a possible cause could be damage to the olfactory neuroepithelium or the central olfactory pathway. ${ }^{7}$ Sudden anosmia is the most common type of olfactory dysfunction in COVID-19 patients and is usually accompanied by nonspecific inflammatory or nasal symptoms. However, isolated sudden anosmia has also been reported in patients with no associated respiratory or gastrointestinal symptoms (as in this case). ${ }^{8)}$

The prognosis of olfactory dysfunction is considered selflimiting in the majority of COVID-19 patients. ${ }^{8)}$ Most (86.8\%) COVID-19 patients with olfactory dysfunction have reported some degree of olfactory recovery. ${ }^{9)}$ Significant improvement has been observed in self-rating the severity of olfactory loss within one week of follow-up. ${ }^{10)}$ However, most studies on olfactory dysfunction in COVID-19 patients are based on subjective discomfort, and the proportion of patients exhibiting actual disturbances and smell recovery is unknown. ${ }^{9)}$

Therefore, objective measurement of olfactory dysfunction without the risk of virus spread is important for the early diagnosis of olfactory dysfunction in COVID-19 patients. A sniffing bead system, a one-off system based on identifying familiar odors, may be helpful (as in this case). It takes lesser than 5 minutes, which is much shorter than conventional olfactory function test, and easy to perform. ${ }^{5)}$ As a screening test, the results correlated with the results of threshold-discriminationidentification score of conventional olfactory function test in patients with/without cognitive impairment. ${ }^{5)}$ Furthermore, the beads used in sniffing bead system are designed for single-use. It can be performed immediately at clinic by doctor during physical examination. These characteristics might en- 
able routine and rapid olfactory function screening for future potential patients with pandemic respiratory disease such as COVID-19.

Few studies have reported the MRI findings of the olfactory area in COVID-19 patients. A study based on 12 patients showed that no patient demonstrated any changes in olfactory bulb volume. Only four of 12 patients demonstrated an abnormally increased olfactory bulb signal on postcontrast $\mathrm{T} 2$ fluid attenuated inversion recovery. ${ }^{11)}$ However, olfactory bulb signal abnormality showed no correlation with the symptoms because only one of the four patients with an olfactory bulb signal abnormality had anosmia. Therefore, imaging studies may not be helpful, and measurement of olfactory function with a verified, population-familiar odorant should be considered for the objective diagnosis of olfactory dysfunction in COVID-19 patients.

Systemic or topical corticosteroids have been routinely used for treating olfactory dysfunction, although the mechanism of their efficacy is unclear. The use of short-term systemic and/or topical steroids is an option in selected patients after careful consideration of their potential risks. ${ }^{12)}$ Other medications, such as zinc sulfate, vitamins, and alpha-lipoic acids, have also been administered. Although various studies have shown that these drugs are beneficial in treating postviral olfactory disorders, a recent meta-analysis study could not show their efficacy in terms of statistical significance. ${ }^{13)}$ Many studies have demonstrated the therapeutic efficacy of olfactory training in postviral olfactory dysfunction. A meta-analysis reported an association between olfactory training and clinically significant improvement in patients with postviral olfactory dysfunction. ${ }^{14)}$ Olfactory training is the only recommended option in managing olfactory dysfunction in COVID-19 patients. ${ }^{15)}$ In our case, the patient underwent olfactory training without any medication and reported recovery of olfactory function after three months. However, there is no obvious evidence for the long-term efficacy of olfactory training in COVID-19 patients. Therefore, long-term follow-up is recommended for patients who have undergone olfactory training due to the absence of early recovery. There are several limitations in this study. First, although subjective olfactory function was recovered by patient's report, additional olfactory function test to demonstrate objective recovery of olfactory dysfunction was not performed. Second, we did not perform additional image study after recovery of olfactory dysfunction of the patient. Comparing images before, and after olfactory recovery might be helpful in evaluating the pathophysiology of olfactory dysfunction in COVID-19 patients.

Olfactory dysfunction is often present in COVID-19 patients, and isolated sudden-onset anosmia can be the sole symptom of COVID-19. Objective measurement of the severity of olfactory dysfunction and long-term follow-up with olfactory training should be performed in such cases.

In conclusion, olfactory dysfunction is common in COVID-19 patients, and isolated sudden-onset anosmia can be the sole symptom of COVID-19. Objective measurement of the severity of olfactory dysfunction such as in our case would be helpful in early and accurate diagnosis of olfactory dysfunction in COVID-19 patients.

\section{Acknowledgments}

This study was approved by the Institutional Review Board of Chung-Ang University College of Medicine and written informed consent was waived.

\section{ORCID}

Hyun Jin Min ～https://orcid.org/0000-0003-3075-1350

\section{REFERENCES}

1) Maniaci A, Iannella G, Vicini C, Pavone P, Nunnari G, Falsaperla $\mathrm{R}$, et al. A case of COVID-19 with late-onset rash and transient loss of taste and smell in a 15 -year-old boy. Am J Case Rep 2020; 21:e925813.

2) Lechien JR, Chiesa-Estomba CM, De Siati DR, Horoi M, Le Bon $\mathrm{SD}$, Rodriguez A, et al. Olfactory and gustatory dysfunctions as a clinical presentation of mild-to-moderate forms of the coronavirus disease (COVID-19): A multicenter European study. Eur Arch Otorhinolaryngol 2020;277(8):2251-61.

3) Won JY, Heo Y, Kim TS, Kim CH, Lee WH. Olfactory and taste dysfunction in patients with asymptomatic and mildly symptomatic COVID-19 in Korea. J Rhinol 2021;28(1):30-5.

4) Suzuki H, Teranishi M, Katayama N, Nakashima T, Sugiura S, Sone M. Relationship between cognitive impairment and olfactory function among older adults with olfactory impairment. Auris Nasus Larynx 2021;48(3):420-7.

5) Min HJ, Kim SM, Han DH, Kim KS. The sniffing bead system, an olfactory dysfunction screening tool for geriatric subjects: A crosssectional study. BMC Geriatr 2021;21(1):54.

6) Ha JG, Kim J, Nam JS, Park JJ, Cho HJ, Yoon JH, et al. Development of a Korean culture-friendly olfactory function test and optimization of a diagnostic cutoff value. Clin Exp Otorhinolaryngol 2020;13(3): 274-84.

7) Mehraeen E, Behnezhad F, Salehi MA, Noori T, Harandi H, SeyedAlinaghi S. Olfactory and gustatory dysfunctions due to the coronavirus disease (COVID-19): A review of current evidence. Eur Arch Otorhinolaryngol 2021;278(2):307-12.

8) Gane SB, Kelly C, Hopkins C. Isolated sudden onset anosmia in COVID-19 infection. A novel syndrome? Rhinology 2020;58(3): 299-301.

9) Liu PY, Jiang RS. Prognosis of olfactory and gustatory dysfunctions in COVID-19 patients: A case series. Clin Case Rep 2020;8(12): 2744-52.

10) Hopkins C, Surda P, Whitehead E, Kumar BN. Early recovery following new onset anosmia during the COVID-19 pandemic - an observational cohort study. J Otolaryngol Head Neck Surg 2020; 
49(1):26.

11) Lin E, Lantos JE, Strauss SB, Phillips CD, Campion TR Jr, Navi $\mathrm{BB}$, et al. Brain imaging of patients with COVID-19: Findings at an academic institution during the height of the outbreak in New York City. AJNR Am J Neuroradiol 2020;41(11):2001-8.

12) Hura N, Xie DX, Choby GW, Schlosser RJ, Orlov CP, Seal SM, et al. Treatment of post-viral olfactory dysfunction: An evidencebased review with recommendations. Int Forum Allergy Rhinol 2020;10(9):1065-86.

13) Miwa $T$, Ikeda K, Ishibashi T, Kobayashi M, Kondo K, Matsuwaki $\mathrm{Y}$, et al. Clinical practice guidelines for the management of olfactory dysfunction - secondary publication. Auris Nasus Larynx 2019; 46(5):653-62.

14) Kattar N, Do TM, Unis GD, Migneron MR, Thomas AJ, McCoul ED. Olfactory training for postviral olfactory dysfunction: Systematic review and meta-analysis. Otolaryngol Head Neck Surg 2021;164(2):244-54.

15) Hopkins C, Alanin M, Philpott C, Harries P, Whitcroft K, Qureishi A, et al. Management of new onset loss of sense of smell during the COVID-19 pandemic - BRS Consensus Guidelines. Clin Otolaryngol 2021;46(1):16-22. 